\title{
Controlling Major Portal Vein Invasion Progression during Lenvatinib Treatment by Carbon-Ion Radiotherapy in Patients with Advanced Hepatocellular Carcinoma
}

\author{
Ryoi Yoshida $^{a}$ Keisuke Koroki ${ }^{a}$ Hirokazu Makishima ${ }^{b}$ \\ Sadahisa Ogasawara ${ }^{a}$ c Takamasa Ishino ${ }^{a}$ Keita Ogawa ${ }^{a}$ \\ Miyuki Nakagawa ${ }^{a}$ Kisako Fujiwara ${ }^{a}$ Hidemi Unozawa ${ }^{a}$ \\ Terunao Iwanaga $^{a}$ Naoto Fujita ${ }^{a}$ Takafumi Sakuma $^{a}$ Hiroaki Kanzaki ${ }^{a}$ \\ Kazufumi Kobayashia, c Naoya Kanogawa ${ }^{a}$ Soichiro Kiyono ${ }^{a}$ \\ Masato Nakamura ${ }^{a}$ Takayuki Kondo ${ }^{a}$ Tomoko Saito $^{a}$ \\ Ryo Nakagawa $^{a}$ Eiichiro Suzuki ${ }^{a}$ Yoshihiko Ooka ${ }^{a}$ Shingo Nakamoto ${ }^{a}$ \\ Akinobu Tawada ${ }^{a}$ d Tetsuhiro Chiba ${ }^{a}$ Makoto Arai ${ }^{a, d}$ Takashi Kaneko $^{b}$ \\ Masaru Wakatsuki ${ }^{b}$ Jun Kato ${ }^{a}$ Hiroshi Tsuji $^{b}$ Naoya Kato $^{a}$ \\ aDepartment of Gastroenterology, Graduate School of Medicine, Chiba University, Chiba, \\ Japan; ${ }^{b}$ National Institutes for Quantum and Radiological Science and Technology QST \\ Hospital, Chiba, Japan; Translational Research and Development Center, Chiba University

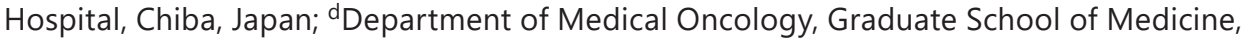 \\ Chiba University, Chiba, Japan
}

Keywords

Carbon-ion radiotherapy $\cdot$ Hepatocellular carcinoma $\cdot$ Macrovascular invasion

\begin{abstract}
Macrovascular invasion (MVI), including portal vein tumor thrombosis (PVTT), is strongly associated with poor prognosis in patients with hepatocellular carcinoma (HCC). While recommended standard treatment for patients with advanced HCC is systemic therapy, various treatment approaches, including resection, transarterial chemoembolization, and radiation, have been empirically suggested to improve prognosis by eliminating or controlling MVI. Herein, we report our experience of a case with advanced HCC where MVI was controlled by carbon-ion radiotherapy (CIRT) while on systemic therapy, resulting in a prolonged survival.
\end{abstract}

Ryoi Yoshida, Keisuke Koroki and Hirokazu Makishima contributed equally to this work. 
A female patient with HCC in her early 60s had multiple intrahepatic lesions (maximum $60 \mathrm{~mm}$ in diameter) with PVTT. The PVTT of this patient had reached the main trunk of the portal vein despite the use of lenvatinib. The other intrahepatic lesions of the patient, except PVTT, had been controlled by lenvatinib. Therefore, hoping to control PVTT, we attempted CIRT. The patient resumed lenvatinib therapy after the irradiation. During lenvatinib re-treatment, no evident progression of PVTT was observed in the patient.

(C) 2021 The Author(s).

Published by S. Karger AG, Basel

\section{Introduction}

Macrovascular invasion (MVI), as represented by portal vein tumor thrombosis (PVTT), is a known poor prognostic factor for advanced hepatocellular carcinoma (HCC) [1]. Although the standard treatment of advanced HCC with MVI is systemic therapy, it has been suggested that surgical resection, transarterial chemoembolization, hepatic arterial infusion chemotherapy (HAIC), and radiation therapy are effective. In clinical practice, those treatments are often selected, with care, alongside systemic therapies [2-4]. Several previous reports have demonstrated that controlling MVI contributes to improved overall survival in patients with HCC [5-10]. Conversely, MVI progression is directly associated with the survival outcome. In particular, the invasion of PVTT into the main trunk of the portal vein (Vp4 according to Japanese classification) also leads to liver function deterioration due to decreased liver blood flow [11, 12].

Particle therapy, including carbon-ion radiotherapy (CIRT), is a radiation therapy modality realizing highly conformal dose distributions and integral dose sparing [13-15]. CIRT has been reported to have a high local control rate and safety in patients with HCC, although most of these reports addressed early to mid-stage HCC that were eligible for curative therapy [16-18]. Recently, while with limited cohort size, there have been a few reports suggesting that particle therapy is effective in patients with advanced HCC with MVI [19-22]. In this report, we present a case of a patient with advanced HCC with PVTT who underwent CIRT aimed at controlling PVTT that progressed during treatment with Lenvatinib, resulting in a prolonged survival.

\section{Case Report/Case Presentation}

A female in her early 60s presented to her primary care physician with fever and abdominal pain. Both abdominal ultrasound and contrast medium-enhanced computed tomography (CE-CT) revealed a 60-mm-diameter hepatic mass at Couinaud's segment VI (Fig. 1a). She was referred to our hospital; we performed tumor biopsy and confirmed the pathological diagnosis of moderately differentiated HCC. The tumor had infiltrated the right branch of the portal vein (Vp3). First, we performed transarterial chemoembolization for tumor control. After tumor shrinkage was achieved, our surgical department performed a posterior hepatic segment resection and cholecystectomy. Two years later, she underwent radiofrequency ablation indicated for recurrence in segment I. In the following year, she had recurrences in segments I and V. For these recurrent intrahepatic lesions, the patient underwent radiofrequency ablation and subsegmental resection for segments I and V, respectively.

After a short time without recurrence, we diagnosed tumor recurrence in segment I and consequent tumor invasion into the Vp4 (Fig. 1b). We performed HAIC to control PVTT. Three courses of low-dose 5 -fluorouracil/cisplatin (FP) therapies had reduced the size of the PVTT;

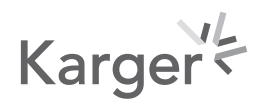




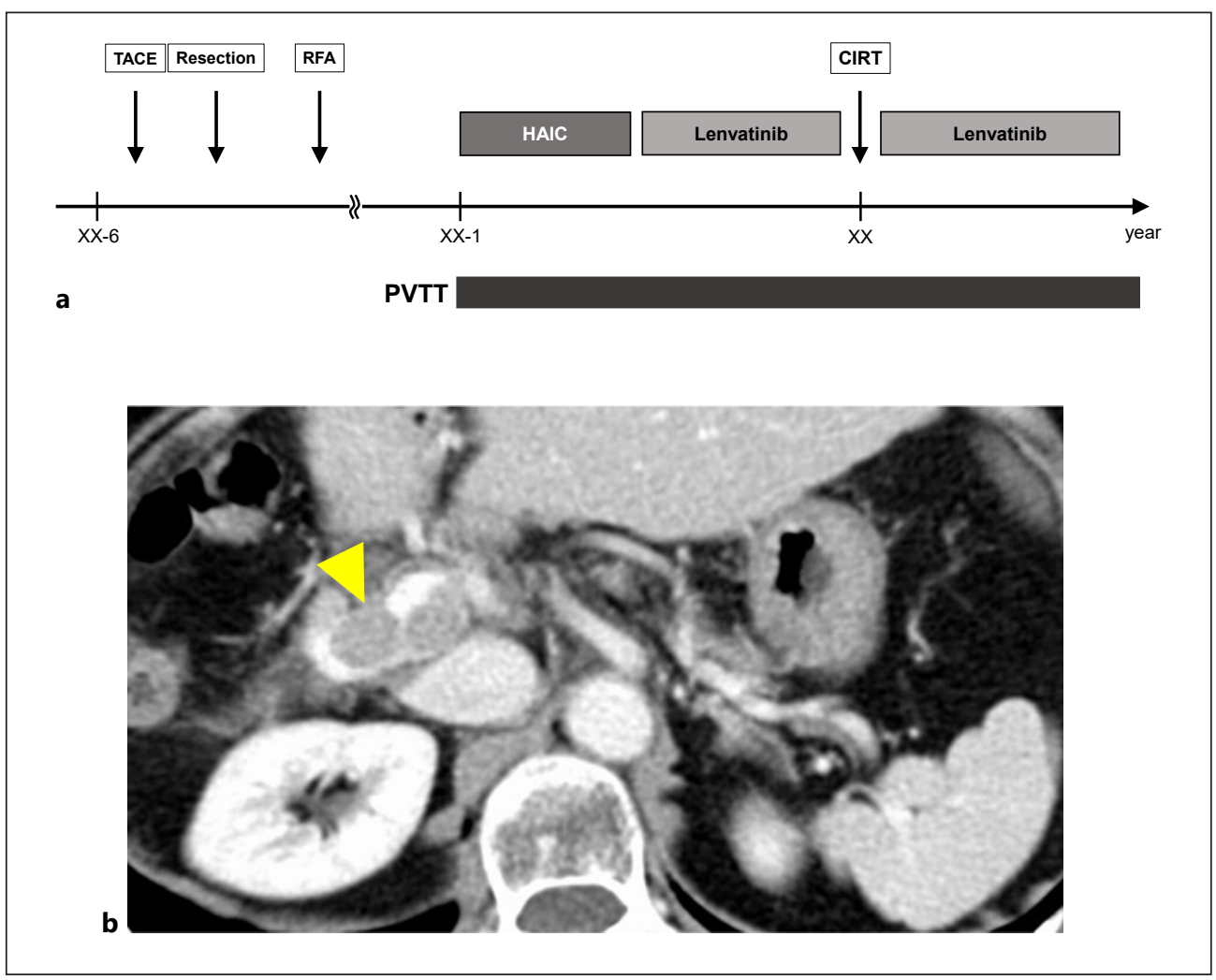

Fig. 1. Clinical course and the PVTT in the main trunk. a Clinical course. The patient was first diagnosed with HCC in the XX-6 and received carbon ion radiotherapy in the XX. b Computed tomography showed a contrast defect in the main trunk of the portal vein due to PVTT and recurrent foci in segment I (Vp4 according to Japanese classification). PVTT, carbon ion radiotherapy; HCC, hepatocellular carcinoma.

however, she developed biloma and was hospitalized for antibiotic therapy. Because of repeated biloma and cholangitis, we performed endoscopic retrograde cholangiopancreatography and placed a metallic stent $(8 \times 80 \mathrm{~mm}$, uncovered $)$ for a bile duct. The biloma and cholangitis were considered to be due to bile duct stenosis caused by HAIC. Therefore, she could not continue HAIC with low-dose FP.

Three months later, the PVTT had extended to the primary left and right branches. We decided to place her on a molecular target agent for lenvatinib ( $8 \mathrm{mg} /$ day). CE-CT 3 months after the administration of lenvatinib showed that the PVTT had progressed to the Vp4 and its primary left and right branches, although there was no visible enlargement of the recurrence site in segment I. After fully informing the patient that lenvatinib, the standard treatment, was ineffective, we suggested her a strategy of performing CIRT to control PVTT. At this time, her liver function was maintained at Child-Pugh score 5A. She then consented to be treated with CIRT (total dose 60 Gy [median RBE-weighted dose] in 4 fractions) (Fig. 2).The patienthad no adverse reactions to the treatment. After irradiation, prothrombin induced by vitamin $\mathrm{K}$ absence or antagonist-II (PIVKA-II) decreased markedly (from 5,569 to $1,609 \mathrm{mAU} / \mathrm{mL}$ ), and CE-CT showed no sign of PVTT progression. Lenvatinib was resumed after CIRT since the intrahepatic lesion was controlled by lenvatinib except for the progression of PVTT. Three months later, there was no evidence of intrahepatic lesion and PVTT progression on CE-CT. Moreover, we observed partial reopening of portal blood flow (Fig. 3). 


\section{Case Reports in Oncology}

Case Rep Oncol 2021;14:1103-1110 \begin{tabular}{l|l}
\hline DOI: $10.1159 / 000517440$ & $\odot 2021$ The Author(s). Published by S. Karger AG, Basel
\end{tabular} www.karger.com/cro

Yoshida et al.: Carbon-Ion Radiotherapy for Major Portal Vein Invasion in Patients with $\mathrm{HCC}$
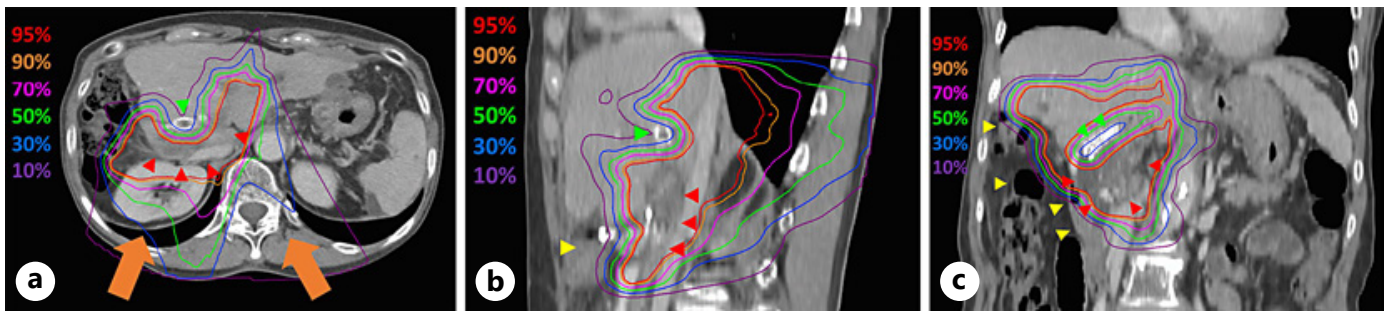

Fig. 2. Dose distribution on computed tomography images. a-c Axial, sagittal, and coronal images of the dose distribution of carbon-ion radiotherapy superimposed on CE-CT. Each isodose line represents the relative dose to the prescribed dose ( $60 \mathrm{~Gy} / 4 \mathrm{Fr}$ ). The red arrowhead shows the tumor, the green arrowhead indicates the metallic stent, and the yellow indicates the gastrointestinal tract; they were required to reduce the exposure dose. The orange arrow shows the beam direction. CE-CT, contrast medium-enhanced computed tomography.

\section{Discussion/Conclusion}

Herein, we demonstrated a patient with advanced HCC in whom successful PVTT control and restoring of portal vein blood flow was achieved by CIRT, even though the PVTT progressed on lenvatinib treatment. Moreover, lenvatinib could be resumed, and the intrahepatic lesion was equally controlled.

Although standard treatment based on a high evidence level is systemic therapy, treatment options for advanced HCC with MVI are diverse in real clinical practice [23-26]. Several guidelines for HCC in Asia, including Japan, recommend treatments other than systemic therapy for advanced HCC with MVI [2-4]. In the present case, HAIC was selected as the first treatment at the time MVI was recognized. Several recent reports have demonstrated that HAIC has survival benefits compared with sorafenib, which had been a standard first-line systemic therapy, until the approval of atezolizumab plus bevacizumab, in patients with advanced disease with MVI without extrahepatic metastasis [27,28]. After discontinuation of HAIC, the patient moved on to lenvatinib, which was the standard systemic therapy like sorafenib at that time [24-26]. Unfortunately, PVTT progressed to the main trunk during lenvatinib treatment. However, in this case, other intrahepatic lesions were controlled by lenvatinib. Therefore, we selected CIRT to control MVI aiming that a further prolongation of the prognosis would be possible if local control of PVTT could be achieved. Since the patient had a history of bile duct stenosis due to HAIC and had a metallic stent inserted, it was necessary to minimize the dose deposited to the metallic stent. Taken together with high local control ability and the ability to produce complex dose distribution, CIRT was sought to be the best treatment choice for this case.

Particle therapy, especially CIRT, is a form of radiotherapy with significant physical and biological advantages over photon irradiation [14,15,29]. Delivering high doses to the tumor while sparing surrounding organs at risk is possible even in larger tumors using particle therapy $[22,30]$.

The present case in which PVTT could be controlled by CIRT suggests the potential of this therapy combined with systemic therapy in patients with advanced HCC with MVI. MVI, including PVTT, is often a strong poor prognostic factor, even more when its control becomes difficult $[11,31,32]$. Several articles suggest particle therapy, both carbon and proton, to be effective in advanced HCC with MVI, with a possibility of cure [19-22] (Table 1). However, high subclinical presence of multiple intrahepatic lesions and/or extrahepatic metastasis associated with advanced HCC with MVI does prevent achieving long time-toprogression. Combination therapy of particle therapy to control the prognosis limiting 
Yoshida et al.: Carbon-Ion Radiotherapy for Major Portal Vein Invasion in Patients with $\mathrm{HCC}$

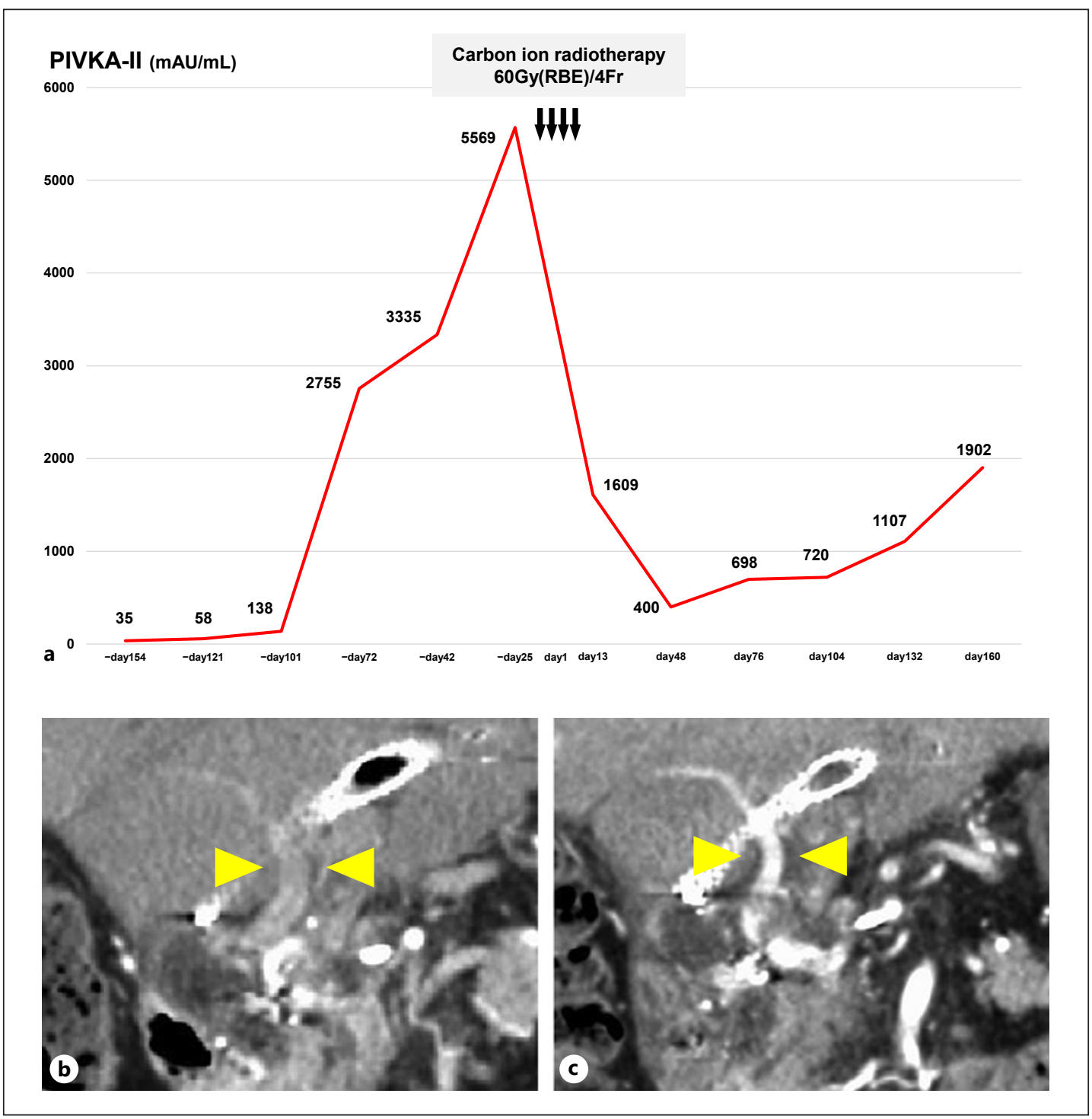

Fig. 3. Changes in the PIVKA-II values and portal vein flow. a After 3 times of low-dose 5-fluorouracil/cisplatin treatment, the PVTT appeared to be shrinking, but a biloma and bile duct stenosis were found. About 2 months after the introduction of lenvatinib, the PVTT developed further and occupied the right and left primary branches. PIVKA-II was increased in proportion to PVTT progression, but it was markedly decreased after CIRT. b Before the CIRT. c Three months after CIRT. Portal vein blood flow has resumed. PIVKA-II, prothrombin induced by vitamin K absence or antagonist-II; PVTT, portal vein tumor thrombosis; CIRT, carbonion radiotherapy.

lesion and pharmacotherapy to control other intra-/extra-hepatic lesions could be an answer to this.

Recently, atezolizumab (anti-PD-L1 inhibitor) plus bevacizumab (anti-VEGF inhibitor) therapy has shown significant improvement in overall survival, progression-free survival, and objective response rate compared to sorafenib in advanced HCC, according to a global phase III trial [23]. Currently, the standard treatment for advanced HCC has been replaced by atezolizumab plus bevacizumab instead of tyrosine kinase inhibitors [33]. In the era of immunotherapy, the effect that radiation therapy has on the tumor microenvironment by being able to convert a cold tumor that has low immunogenicity and is poorly infiltrated with 
Table 1. Lists of past reports of HCC with MVI treated with proton beam therapy and CIRT

\begin{tabular}{lllllll}
\hline Author & $n$ & Therapy & RT total dose & Fx & Location & LPFS or LC rates \\
\hline Hata et al. [19] & 12 & Proton beam & 55.0 GyE (50 72) & 10-22 Fr & Vp3, Vp4 & 67\% (2-year) \\
Sugahara et al. [20] & 35 & Proton beam & 72.6 GyE (55.0 77.0) & 22 Fr & Vp3, Vp4 & 46\% (2-year) \\
Lee et al. [21] & 27 & Proton beam & $50 \sim 66 \mathrm{GyE}$ & $20-22 \mathrm{Fr}$ & Vp3, Vp4 & $64.2 \%$ (2-year) \\
Shiba et al. [22] & 11 & Carbon ion & 52.8 60 GyE & 4 or 12 Fr & Vv3, Vp4 & 78\% (3-year) \\
\hline
\end{tabular}

HCC, hepatocellular carcinoma; PVTT, portal vein tumor thrombosis; RT, radiotherapy; Fx, fraction; LPFS, local progressive-free survival; LC, local control; MVI, macrovascular invasion; CIRT, carbon-ion radiotherapy.

immune cells to an immune-reactive hot tumor that is well infiltrated by immune cells has been the main focus of radiation therapy [34-36]. Specifically, radiation therapy has the potential to enhance the effect of immune checkpoint inhibitors. In advanced HCC with MVI, irradiating the tumor with MVI by particle radiation therapy and combining it with an immune checkpoint inhibitor may provide synergistic treatment effects based on 2 abilities of particle radiation therapy, that is, localized tumor killing and tumor microenvironment modification. We believe this would dramatically improve the survival outcomes in patients with advanced HCC with MVI, which has the worst prognosis of HCC.

In conclusion, we experienced a patient with advanced HCC with MVI in whom PVTT that progressed while on lenvatinib treatment was controlled by CIRT. Combining CIRT with systemic therapy may be a treatment option in patients with advanced HCC with MVI.

\section{Acknowledgement}

The authors would like to thank Enago (www.enago.jp) for the English language review.

\section{Statement of Ethics}

This research was conducted ethically in accordance with the World Medical Association Declaration of Helsinki. The patient is deceased. The informed consent for publication was obtained from the patient's family.

\section{Conflict of Interest Statement}

Sadahisa Ogasawara received grant support from Eisai, Bayer, and Eli Lilly, advisory fees from Eisai, Bayer, MSD, AstraZeneca, and Eli Lilly, and honoraria from Eisai, Bayer, MSD, AstraZeneca, and Eli Lilly. Yoshihiko Ooka received honoraria from Eisai. Naoya Kato received grant support from Eisai, Bayer, Takeda, and Eli Lilly, advisory fees from Eisai, Bayer, and Eli Lilly, and honoraria from Eisai, Bayer, and Eli Lilly. The other authors of this study indicated that they had nothing to declare regarding funding or conflict of interest with respect to this study.

\section{Funding Sources}

This research received no external funding.

\section{Karger'}




\section{Case Reports in Oncology}

\begin{tabular}{l|l}
\hline Case Rep Oncol 2021;14:1103-1110 \\
\hline DOI: 10.1159/000517440 & $\begin{array}{l}\text { ○ 2021 The Author(s). Published by S. Karger AG, Basel } \\
\text { www.karger.com/cro }\end{array}$ \\
\hline
\end{tabular}

Yoshida et al.: Carbon-Ion Radiotherapy for Major Portal Vein Invasion in Patients with $\mathrm{HCC}$

\section{Author Contributions}

Conceptualization, Sadahisa Ogasawara; methodology, Sadahisa Ogasawara; investigation, Ryoi Yoshida, Keisuke Koroki, Takamasa Ishino, Keita Ogawa, Miyuki Nakagawa, Kisako Fujiwara, Hidemi Unozawa, Terunao Iwanaga, Naoto Fujita, Takafumi Sakuma, Hiroaki Kanzaki, Kazufumi Kobayashi, Naoya Kanogawa, Soichiro Kiyono, Masato Nakamura, Takayuki Kondo, Tomoko Saito, Ryo Nakagawa, Eiichiro Suzuki, Yoshihiko Ooka, Shingo Nakamoto, Akinobu Tawada, Tetsuhiro Chiba, Makoto Arai, Takashi Kaneko; data curation, Ryoi Yoshida, Keisuke Koroki; writing - original draft preparation, Ryoi Yoshida, Keisuke Koroki, Hirokazu Makihsima; writing - review and editing, Sadahisa Ogasawara, Hirokazu Makishima; supervision, Masaru Wakatsuki, Hiroshi Tsuji, Jun Kato, Naoya Kato; project administration, Naoya Kato.

\section{References}

1 Finn RS, Zhu AX, Farah W, Almasri J, Zaiem F, Prokop LJ, et al. Therapies for advanced stage hepatocellular carcinoma with macrovascular invasion or metastatic disease: a systematic review and meta-analysis. Hepatology. 2018 Jan;67(1):422-35.

2 Kokudo N, Takemura N, Hasegawa K, Takayama T, Kubo S, Shimada M, et al. Clinical practice guidelines for hepatocellular carcinoma: the Japan Society of Hepatology 2017 (4th JSH-HCC guidelines) 2019 update. Hepatol Res. 2019 Oct;49(10):1109-13.

3 Omata M, Cheng AL, Kokudo N, Kudo M, Lee JM, Jia J, et al. Asia-Pacific clinical practice guidelines on the management of hepatocellular carcinoma: a 2017 update. Hepatol Int. 2017 Jul;11(4):317-70.

4 Galle PR, Forner A, Llovet JM, Mazzaferro V, Piscaglia F, Raoul J-L, et al. EASL clinical practice guidelines: management of hepatocellular carcinoma. J Hepatol. 2018 Jul;69(1):182-236.

5 Kokudo T, Hasegawa K, Matsuyama Y, Takayama T, Izumi N, Kadoya M, et al. Survival benefit of liver resection for hepatocellular carcinoma associated with portal vein invasion. J Hepatol. 2016 Nov;65(5): 938-43.

6 Chung GE, Lee JH, Kim HY, Hwang SY, Kim JS, Chung JW, et al. Transarterial chemoembolization can be safely performed in patients with hepatocellular carcinoma invading the main portal vein and may improve the overall survival. Radiology. 2011 Feb;258(2):627-34.

7 Nouso K, Miyahara K, Uchida D, Kuwaki K, Izumi N, Omata M, et al. Effect of hepatic arterial infusion chemotherapy of 5-fluorouracil and cisplatin for advanced hepatocellular carcinoma in the Nationwide Survey of Primary Liver Cancer in Japan. Br J Cancer. 2013 Oct;109(7):1904-7.

8 Moriguchi M, Aramaki T, Nishiofuku H, Sato R, Asakura K, Yamaguchi K, et al. Sorafenib versus hepatic arterial infusion chemotherapy as initial treatment for hepatocellular carcinoma with advanced portal vein tumor thrombosis. Liver Cancer. 2017 Nov;6(4):275-86.

9 Nakazawa T, Hidaka H, Shibuya A, Okuwaki Y, Tanaka Y, Takada J, et al. Overall survival in response to sorafenib versus radiotherapy in unresectable hepatocellular carcinoma with major portal vein tumor thrombosis: propensity score analysis. BMC Gastroenterol. 2014 May 3;14:84.

10 Kim N, You MW. Hepatocellular carcinoma and macrovascular tumor thrombosis: treatment outcomes and prognostic factors for survival. Jpn J Radiol. 2019 Nov;37(11):781-92.

11 Llovet JM, Bustamante J, Castells A, Vilana R, Ayuso MDC, Sala M, et al. Natural history of untreated nonsurgical hepatocellular carcinoma: rationale for the design and evaluation of therapeutic trials. Hepatology. 1999 Jan; 29(1):62-7.

12 Chan SL, Mo FK, Johnson PJ, Liem GS, Chan TC, Poon MC, et al. Prospective validation of the Chinese University Prognostic Index and comparison with other staging systems for hepatocellular carcinoma in an Asian population. J Gastroenterol Hepatol. 2011 Feb;26(2):340-7.

13 Weyrather WK, Debus J. Particle beams for cancer therapy. Clin Oncol (R Coll Radiol). 2003 Feb;15(1):S23-8.

14 Kanai T, Furusawa Y, Fukutsu K, Itsukaichi H, Eguchi-Kasai K, Ohara H. Irradiation of mixed beam and design of spread-out bragg peak for heavy-ion radiotherapy. Radiat Res. 1997;147:78-85.

15 Pedroni E, Bacher R, Blattmann H, Böhringer T, Coray A, Lomax A, et al. The 200-mev proton therapy project at the paul scherrer institute: conceptual design and practical realization. Med Phys. 1995 Jan; 22(1):37-53.

16 Kasuya G, Kato H, Yasuda S, Tsuji H, Yamada S, Haruyama Y, et al. Progressive hypofractionated carbon-ion radiotherapy for hepatocellular carcinoma: combined analyses of 2 prospective trials. Cancer. 2017 Oct;123(20): 3955-65.

17 Shiba S, Abe T, Shibuya K, Katoh H, Koyama Y, Shimada H, et al. Carbon ion radiotherapy for 80 years or older patients with hepatocellular carcinoma. BMC Cancer. 2017 Nov;17(1):721. 


\section{Case Reports in Oncology}

\begin{tabular}{l|l}
\hline Case Rep Oncol 2021;14:1103-1110 \\
\hline DOI: 10.1159/000517440 & $\begin{array}{l}\text { ○ 2021 The Author(s). Published by S. Karger AG, Basel } \\
\text { www.karger.com/cro }\end{array}$ \\
\hline
\end{tabular}

Yoshida et al.: Carbon-Ion Radiotherapy for Major Portal Vein Invasion in Patients with $\mathrm{HCC}$

18 Shibuya K, Ohno T, Katoh H, Okamoto M, Shiba S, Koyama Y, et al. A feasibility study of high-dose hypofractionated carbon ion radiation therapy using four fractions for localized hepatocellular carcinoma measuring $3 \mathrm{~cm}$ or larger. Radiother Oncol. 2019 Mar;132:230-5.

19 Hata M, Tokuuye K, Sugahara S, Kagei K, Igaki H, Hashimoto T, et al. Proton beam therapy for hepatocellular carcinoma with portal vein tumor thrombus. Cancer. 2005 Aug;104(4):794-801.

20 Sugahara S, Nakayama H, Fukuda K, Mizumoto M, Tokita M, Abei M, et al. Proton-beam therapy for hepatocellular carcinoma associated with portal vein tumor thrombosis. Strahlenther Onkol. 2009 Dec;185(12):782-8.

21 Lee SU, Park JW, Kim TH, Kim YJ, Woo SM, Koh YH, et al. Effectiveness and safety of proton beam therapy for advanced hepatocellular carcinoma with portal vein tumor thrombosis. Strahlenther Onkol. 2014 Sep; 190(9): 806-14.

22 Shiba S, Shibuya K, Okamoto M, Okazaki S, Komatsu S, Kubota Y, et al. Clinical impact of hypofractionated carbon ion radiotherapy on locally advanced hepatocellular carcinoma. Radiat Oncol. 2020 Aug;15(1):195.

23 Finn RS, Qin S, Ikeda M, Galle PR, Ducreux M, Kim TY, et al. Atezolizumab plus bevacizumab in unresectable hepatocellular carcinoma. N Engl J Med. 2020 May;382(20):1894-905.

24 Llovet JM, Ricci S, Mazzaferro V, Hilgard P, Gane E, Blanc JF, et al. Sorafenib in advanced hepatocellular carcinoma. N Engl J Med. 2008 Jul;359(4):378-90.

25 Cheng AL, Kang YK, Chen Z, Tsao CJ, Qin S, Kim JS, et al. Efficacy and safety of sorafenib in patients in the AsiaPacific region with advanced hepatocellular carcinoma: a phase III randomized, double-blind, placebocontrolled trial. Lancet Oncol. 2009 Jan;10(1):25-34.

26 Kudo M, Finn RS, Qin S, Han KH, Ikeda K, Piscaglia F, et al. Lenvatinib versus sorafenib in first-line treatment of patients with unresectable hepatocellular carcinoma: a randomised phase 3 non-inferiority trial. Lancet. 2018 Mar;391(10126):1163-73.

27 Ueshima K, Ogasawara S, Ikeda M, Yasui Y, Terashima T, Yamashita T, et al. Hepatic arterial infusion chemotherapy versus sorafenib in patients with advanced hepatocellular carcinoma. Liver Cancer. 2020 Sep; 9(5): 583-95.

28 Iwamoto H, Niizeki T, Nagamatsu H, Ueshima K, Nomura T, Kuzuya T, et al. Survival benefit of hepatic arterial infusion chemotherapy over sorafenib in the treatment of locally progressed hepatocellular carcinoma. Cancers. 2021 Feb;13(4):646.

29 Kanai T, Endo M, Minohara S, Miyahara N, Koyama-ito H, Tomura H, et al. Biophysical characteristics of himac clinical irradiation system for heavy-ion radiation therapy. Int J Radiat Oncol Biol Phys. 1999 Apr;44(1):20110.

30 Mizumoto M, Okumura T, Hashimoto T, Fukuda K, Oshiro Y, Fukumitsu N, et al. Proton beam therapy for hepatocellular carcinoma: a comparison of three treatment protocols. Int J Radiat Oncol Biol Phys. 2011 Nov;81(4): 1039-45.

31 Yu AS, Keeffe EB. Management of hepatocellular carcinoma. Rev Gastroenterol Disord. 2003;3(1):8-24.

32 Lee HS, Kim JS, Choi IJ, Chung JW, Park JH, Kim CY. The safety and efficacy of transcatheter arterial chemoembolization in the treatment of patients with hepatocellular carcinoma and main portal vein obstruction. A prospective controlled study. Cancer. 1997 Jun; 79(11):2087-94.

33 Llovet JM, Villanueva A, Marrero JA, Schwartz M, Meyer T, Galle PR, et al. Trial design and endpoints in hepatocellular carcinoma: AASLD consensus conference. Hepatology. 2021 Jan;73(Suppl 1):158-91.

34 Chakraborty M, Abrams SI, Coleman CN, Camphausen K, Schlom J, Hodge JW. External beam radiation of tumors alters phenotype of tumor cells to render them susceptible to vaccine-mediated T-cell killing. Cancer Res. 2004 Jun;64(12):4328-37.

35 Deng L, Liang H, Burnette B, Beckett M, Darga T, Weichselbaum RR, et al. Irradiation and anti-PD-L1 treatment synergistically promote antitumor immunity in mice. J Clin Invest. 2014 Feb;124(2):687-95.

36 Lugade AA, Moran JP, Gerber SA, Rose RC, Frelinger JG, Lord EM. Local radiation therapy of B16 melanoma tumors increases the generation of tumor antigen-specific effector cells that traffic to the tumor. J Immunol. 2005 Jun;174(12):7516-23. 